\title{
Matrix Metalloproteinase-2 Degrades Fibrillin-1 and Fibrillin-2 of Oxytalan Fibers in the Human Eye and Periodontal Ligaments In Vitro
}

\author{
Megumi Kawagoe $^{1}$, Eichi Tsuruga ${ }^{3}$, Kyoko Oka², Yoshihiko Sawa ${ }^{3}$ and Hiroyuki Ishikawa ${ }^{1}$ \\ ${ }^{I}$ Section of Orthodontics, Department of Oral Growth and Development, Division of Clinical Dentistry, Fukuoka Dental College, \\ 2-15-1 Tamura, Sawara-ku, Fukuoka 814-0193, Japan, ${ }^{2}$ Section of Pediatric Dentistry, Department of Oral Growth and \\ Development, Division of Clinical Dentistry, Fukuoka Dental College, 2-15-1 Tamura, Sawara-ku, Fukuoka 814-0193, \\ Japan and ${ }^{3}$ Section of Functional Structure, Department of Morphological Biology, Division of Biomedical Sciences, \\ Fukuoka Dental College, 2-15-1 Tamura, Sawara-ku, Fukuoka 814-0193, Japan
}

Received July 24, 2013; accepted September 18, 2013; published online October 23, 2013

\begin{abstract}
Oxytalan fibers are distributed in the eye and periodontal ligaments (PDL). The ciliary zonule, known as Zinn's zonule, in the eye is composed of oxytalan fibers, which are bundles of microfibrils consisting mainly of fibrillin-1 and fibrillin-2. As turnover of oxytalan fibers is slow during life, their degradation mechanism remains unclarified. This study was performed to examine degradation pattern of fibrillin-1 and fibrillin-2 by experimental MMP activation. We cultured human non-pigmented ciliary epithelial cells (HNPCEC) and PDL fibroblasts for 7 days, then treated them with concanavalin $A$ to activate matrix metalloproteinase (MMP)-2, and examined the degradation of fibrillin-1 and fibrillin-2 for $72 \mathrm{hr}$ using immunofluorescence. At 7 days of HNPCEC culture, fibrillin-1-positive fibers were observed, some of which merged with fibrillin-2. After MMP-2 activation, fibrillin-1-positive fibers became thin and disappeared by $72 \mathrm{hr}$, while fibrillin-2-positive fibers disappeared almost completely within $24 \mathrm{hr}$. At 7 days of PDL fibroblast culture, fibrillin-1-positive fibers were mostly merged with fibrillin-2. After MMP-2 activation, fibrillin-1-positive fibers became thin by $24 \mathrm{hr}$ and had almost disappeared by $48 \mathrm{hr}$, while fibrillin-2-positive fibers decreased constantly after $24 \mathrm{hr}$. A MMP-2 inhibitor completely suppressed these degradations. These results suggest that the patterns of fibrillin-1 and fibrillin-2 degradation differ between the eye and the PDL, possibly reflecting the sensitivity of fibrillin-1 and fibrillin-2 of each type of oxytalan fiber against MMP-2.
\end{abstract}

Key words: fibrillin, microfibrils, oxytalan fibers, ciliary zonule

\section{Introduction}

Elastic system fibers are the major component of the extracellular matrix, providing tissues with elasticity. Elastic system fibers are composed of two components: elastin and microfibrils [17]. Depending upon the relative proportions and morphological arrangement of these two components, three types of elastic system fiber can be

Correspondence to: Eichi Tsuruga, Section of Functional Structure, Department of Morphological Biology, Division of Biomedical Sciences, Fukuoka Dental College, 2-15-1 Tamura, Sawara-ku, Fukuoka 814-0193, Japan

E-mail: tsuru@college.fdcnet.ac.jp identified: elastic, elaunin, and oxytalan fibers [2]. Elastic fibers contain a relatively large amount of elastin, whereas elaunin fibers contain very little. By contrast, oxytalan fibers are bundles of pure microfibrils. Oxytalan fibers were first described in periodontal ligaments (PDL) by Fullmer and Lillie [7]. Microfibrils are composed mainly of fibrillin-1 and fibrillin-2 molecules, which are cross-linked by transglutaminase homo- and/or heterotypically [14].

In the human body, pure oxytalan fibers exist in the eye as well as in the PDL. In the eye, the ciliary zonule, also known as Zinn's zonule, is composed of pure microfibrils [19]. The ciliary zonule connects the lens to the ciliary body, in order to control the thickness of the lens for focusing. Proteomics analysis has shown that the human 
ciliary zonule contains fibrillin-1 and microfibril-associated glycoprotein-1 among microfibril-associated molecules [3]. In the guinea pig, non-pigmented ciliary epithelial cells in the ciliary body express fibrillin- 1 mRNA and the ciliary zonule is labeled by fibrillin- 1 antibody, as revealed by immunofluorescence [8]. Recently, we showed that human non-pigmented ciliary epithelial cells (HNPCEC) express fibrillin-2 as well as fibrillin-1, and that fibrillin-2 may have a role in the formation of thick oxytalan fibers in vitro [31]. More recently, mouse non-pigmented ciliary epithelial cells have been shown to express both fibrillin-1 and fibrillin-2 mRNA, and the ciliary zonule is labeled for both fibrillins by in situ hybridization and immunofluorescence [24], being consistent with our result [31].

The oxytalan fibers in PDL are arranged in a vertically-oriented interlacing network enclosing the molar root apex [25]. Using immunohistocheminal analysis in the rat [26] and immunoelectron analysis in the monkey [23], it has been demonstrated that oxytalan fibers in the PDL are associated with fibrillin-1 and fibrillin-2. Our series of studies have shown that human PDL fibroblasts express both fibrillins and produce a network of oxytalan fibers associated with them [27-29]. In the PDL as well the eye, oxytalan fibers maintain homeostasis as part of the extracellular matrix, which is dependent on a balance between production and degradation. However, since it is not easy to study oxytalan fibers by biochemical assay and their turnover rate is very slow [17], the mechanism responsible for their degradation remains unclear.

The matrix metalloproteinases (MMPs) are a major group of enzymes responsible for the degradation of extracellular matrices [30]. MMPs are secreted as latent forms (proMMPs) and require activation, possibly by cleavage of the N-terminal prodomain. Among the MMPs, MMP-2 is the principal enzyme working under physiological conditions [15]. HNPCEC and PDL fibroblasts express MMP-2 and secrete it into the matrices in cell culture. Both fibrillin-1 and fibrillin-2 are substrates for MMP-2 [1, 10]. Therefore, our present culture system would seem suitable for examining the process of degradation of fibrillin-1 and fibrillin-2 by MMP-2. Sato et al. have demonstrated that concanavalin A (ConA) converts proMMP-2 to active MMP-2 in culture [22]. Therefore, we cultured HNPCEC and PDL fibroblasts for 7 days to form a fibrillin- 1 and fibrillin-2 network, and then, using immunofluorescence, we compared the degradation of each of fibrillin-1 and fibrillin-2 by ConA-activated MMP-2.

\section{Materials and Methods}

\section{Cell culture and treatment}

The protocol for these experiments was reviewed and approved by the Fukuoka Dental College Research Ethics Committee, and informed consent was obtained from the tissue donors.

PDL fibroblasts were isolated from three different donors and cultured, as described previously [18]. HNPCEC were purchased from ScienCell Research Laboratories (Carlsbad, CA, USA) and cultured in Dulbecco's modified Eagle Medium (DMEM) (Invitrogen, Grand Island, NY, USA). Culture media for both PDL fibroblasts and HNPCEC were supplemented with $10 \%$ newborn calf serum (NCS; Invitrogen) and 100 units $/ \mathrm{ml}$ penicillin and $100 \mu \mathrm{g} / \mathrm{ml}$ streptomycin (Roche Diagnostics, Mannheim, Germany) at $37^{\circ} \mathrm{C}$ in humidified air containing $5 \% \mathrm{CO}_{2}$. When cells reached confluence, they were harvested with $0.025 \%$ trypsin (Invitrogen) in PBS, and transferred to plastic culture dishes at a $1: 4$ split ratio. For experiments, the cells were trypsinized and seeded at $1 \times 10^{6}$ cells $/ \mathrm{ml}$ per 35-mm culture dish (Corning Inc., Corning, NY, USA). The PDL fibroblasts and HNPCEC were found to be confluent after $72 \mathrm{hr}$, and cultured for 7 days for this experiment. The PDL fibroblasts were from three different donors, and used from the 3rd to 6th passages.

After 7 days, the cultures were supplemented with 0 $50 \mathrm{ng} / \mathrm{ml}$ ConA (Sigma-Aldrich, St. Louis, MO, USA) (this time point being set as $0 \mathrm{hr}$ after MMP-2 activation) and/or $2.5 \mu \mathrm{M}$ MMP-2 inhibitor I (EMD, Millipore, Billerica, MA, USA), and immunofluorescence analyses were carried out at $0,24,48$, and $72 \mathrm{hr}$. A stock solution of the MMP-2 inhibitor I was dissolved in dimethyl sulfoxide (DMSO), and subsequently diluted with DMEM. The concentration of MMP-2 inhibitor I employed had been determined in previous culture experiments [9]. Control cultures contained an equivalent concentration of DMSO in DMEM, instead of MMP-2 inhibitor I. The final concentration of DMSO did not exceed $0.2 \%(\mathrm{v} / \mathrm{v})$.

\section{Gelatin zymography}

Gelatinase activities in the cell lysates at $6 \mathrm{hr}$ after ConA treatment were determined using SDS-polyacrylamide gel electrophoresis zymography. Cell lysates $(10 \mu \mathrm{g})$ were electrophoresed without reduction on SDSpolyacrylamide gels prepared with $10 \%$ acrylamide containing $0.1 \%$ gelatin. The SDS was removed by a $1-\mathrm{hr}$ incubation in $2.5 \%$ Triton $\mathrm{X}-100$, and the gels were then incubated in $30 \mathrm{mM}$ Tris- $\mathrm{HCl}(\mathrm{pH} \mathrm{7.4),} 200 \mathrm{mM} \mathrm{NaCl}$, $5 \mathrm{mM} \mathrm{CaCl}_{2}$, and $1 \mathrm{mM} \mathrm{ZnCl}_{2}$, at $37^{\circ} \mathrm{C}$ for $24 \mathrm{hr}$ prior to staining with Coomassie Blue (Bio-Rad, Hertfordshire, UK). Enzyme activity was visualized as zones of gelatin clearance within the gels.

\section{Immunofluorescence}

At $0,24,48$ and $72 \mathrm{hr}$ of culture treatment, HNPCEC and PDL fibroblasts were fixed in ice-cold $4 \%$ paraformaldehyde for $15 \mathrm{~min}$, followed by washing with PBS. Nonspecific immunoreactivity was blocked with $1 \%$ goat serum (Sigma-Aldrich) in PBS for $1 \mathrm{hr}$ at room temperature. The cell matrix layers were then incubated for $2 \mathrm{hr}$ at room temperature with the appropriate primary antibodies (clone 11C1.3, monoclonal antibody against human fibrillin-1 diluted 1:1000; Thermo Fisher Scientific. Anatomical Pathology, Fremont, CA, USA, rabbit antibody against human fibrillin-2 diluted 1:1000; Elastin Products Co., 
Owensville, MO, USA). Controls included the use of preimmune normal mouse and rabbit IgG for incubation with the primary antibody. After being rinsed in PBS, the cells were incubated with Alexa Fluor ${ }^{\circledR}$ 488-labeled goat anti-mouse IgG antibody or Alexa Fluor ${ }^{\circledR}$ 568-labeled goat anti-rabbit IgG antibody (Molecular Probes, Eugene, OR, USA), diluted 1:2000 with blocking buffer, for $1 \mathrm{hr}$ at room temperature. After a final wash, the cells were viewed using a confocal microscope (MRC-1024; Bio-Rad).

Planimetry analysis of the positive staining signals was performed using the Image $\mathrm{J}$ program (National Institutes of Health, Bethesda, MD, USA), as described previously [32]. The area of positive staining divided by the total area was calculated. Each value presented is expressed as the mean \pm standard deviation (SD), and all quantitative results represent at least four independent analyses.

\section{Results}

\section{ConA-induced MMP-2 activation}

When HNPCEC and PDL fibroblasts were treated with ConA (0-50 ng/ml), spontaneously produced $72-\mathrm{kDa}$ pro-MMP-2 was converted to 62-kDa active MMP-2 with the appearance of a $64-\mathrm{kDa}$ intermediate form in a dosedependent manner (Fig. 1A, B). The 62-kDa active MMP2 was clearly confirmed when the cells were treated with $50 \mathrm{ng} / \mathrm{ml} \mathrm{ConA}$ after $6 \mathrm{hr}$. This activation continued for at least 72 hr (Fig. 1C).

\section{Degradation of fibrillin-1 and -2 in HNPCEC and PDL fibroblasts by active MMP-2}

Previously, we had confirmed that HNPCEC and PDL fibroblasts produce oxytalan fibers consisting of fibrillin-1 and fibrillin-2 [29, 31]. We had also shown that at 7 days of HNPCEC culture, thick fibrillin-1-positive fibers occupied most of the area in which fibrillin-2 positivity was detected [31]. Therefore, we activated MMP-2 and observed the subsequent degradation of fibrillin-1 and fibrillin-2 using immunofluorescence in HNPCEC culture (Fig. 2A). At $0 \mathrm{hr}$ after MMP-2 activation (control), fibers positive for fibrillin-2 were detected on the thick fibrillin-1-positive fibers, as had been observed previously. At around $72 \mathrm{hr}$, fibrillin-1 positive thick fibers gradually became thin and finally disappeared. At the same time, Image $J$ area analysis demonstrated an almost constant decrease of the fibrillin-1positive area between 0 and $72 \mathrm{hr}$ (Fig. 2B). On the other hand, fibrillin-2-positive thick fibers decreased rapidly by $24 \mathrm{hr}$ and disappeared by $72 \mathrm{hr}$ (Fig. 2A), in agreement with area analysis demonstrating a reduction of the fibrillin2-positive area to almost zero by $24 \mathrm{hr}$ after MMP-2 activation (Fig. 2B).

At 7 days of PDL fibroblast culture, fibrillin-2 was labeled on a proportion of fibrillin-1-immunolabeled fibers. Similarly, after activation of MMP-2, we observed the degradation of fibrillin-1 and fibrillin-2 using immunofluorescence in PDL fibroblasts culture (Fig. 3A). At 0 hr after MMP-2 activation (control), fibers positive for fibrillin-2 were detected on the fibrillin-1-positive fibers, as had been observed in our previous study [29]. Fibrillin-1positive fibers rapidly became thin by $24 \mathrm{hr}$, and had almost disappeared by $48 \mathrm{hr}$. At the same time, Image $\mathrm{J}$ area analysis showed that MMP-2 activation reduced the level of fibrillin- 1 to $10 \%$ of the control by $24 \mathrm{hr}$ and to $0 \%$ by $48 \mathrm{hr}$ (Fig. 3B). On the other hand, fibrillin-2-positive fibers showed no apparent degradation by $24 \mathrm{hr}$ and decreased constantly after $24 \mathrm{hr}$, but were still clearly detectable at 72
(A)

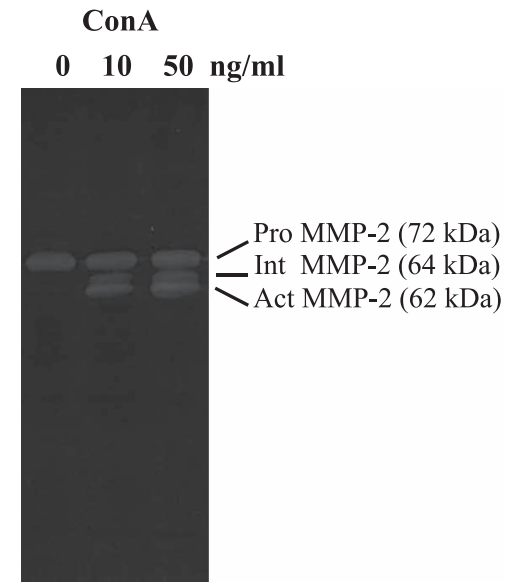

(B)

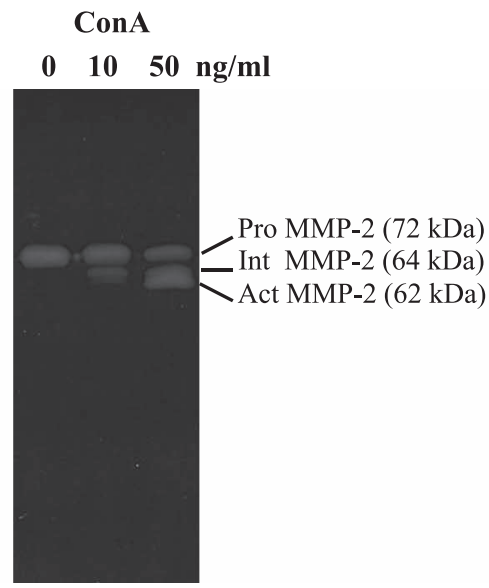

(C)

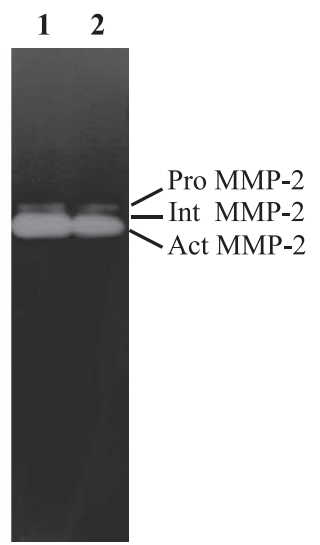

Fig. 1. ConA-induced pro-MMP-2 activation. Human non-pigmented ciliary epithelial cells (A) and periodontal ligaments fibroblasts (B) were cultured for 7 days, and then various concentrations $(0,10,50 \mathrm{ng} / \mathrm{ml})$ of ConA were added to the medium for an additional $72 \mathrm{hr}$. Cell lysates obtained at $6 \mathrm{hr}$ after addition of ConA were analyzed by zymography. Harvested samples from non-pigmented ciliary epithelial cells (lane 1) and periodontal ligament fibroblast (lane 2) obtained at $72 \mathrm{hr}$ were similarly subjected to gelatin zymography (C). Pro-MMP-2: latent-MMP-2, 72 kDa; Int MMP-2: Intermediate-MMP-2, 64 kDa; Act MMP-2: Active-MMP-2, 62 kDa 
(A)

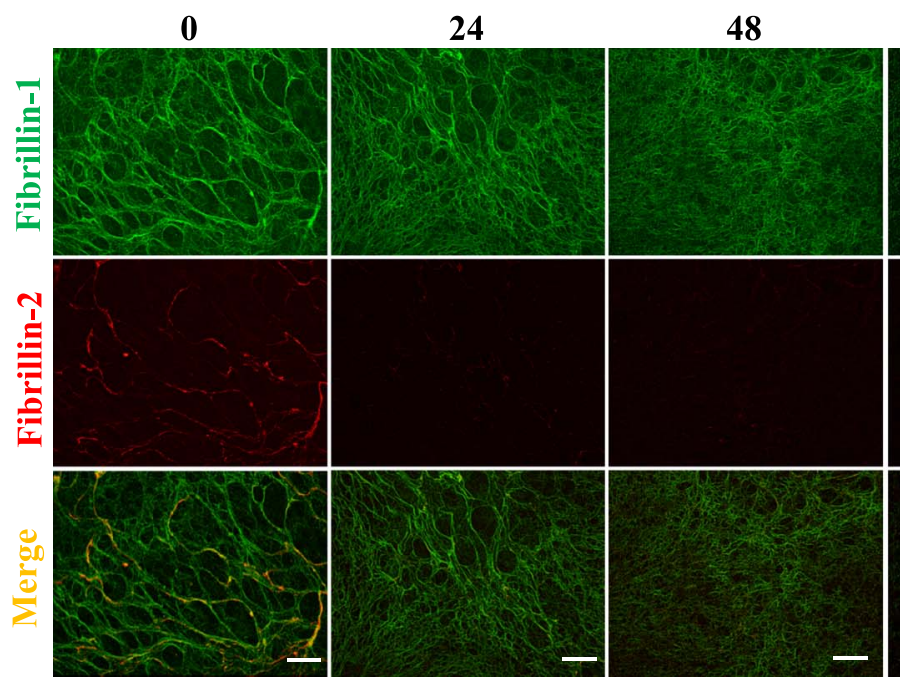

(B)

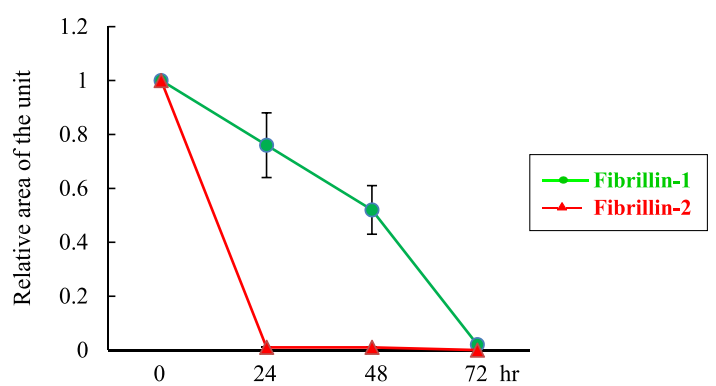

Fig. 2. Degradation of fibrillin-1 and fibrillin-2 by active MMP-2. (A) Double immunofluorescence for fibrillin-1 (upper panels) and fibrillin-2 (middle panels) in cultures of human non-pigmented ciliary epithelial cells. Human non-pigmented ciliary epithelial cells were cultured for 7 days, and then $50 \mathrm{ng} / \mathrm{ml}$ ConA was added to the medium for an additional $72 \mathrm{hr}$. Cells were then labeled simultaneously for fibrillin-1 (green) (upper panels), fibrillin-2 (red) (middle panels), and superimposition of both labels (lower panels) at 0, 24, 48 and $72 \mathrm{hr}$. Bar=20 $\mu \mathrm{m}$. (B) Planimetry analysis of the positive staining signals was performed using the Image J program. The relative area of positive signals per unit area at $0 \mathrm{hr}$ was calculated using Image $J$, with the control set as 1 . Data represent the mean \pm S.D. (standard deviation) of four independent experimental determinations.

hr (Fig. 3A). As supported by immunofluorescence observations, the fibrillin-2-positive area decreased with time after $24 \mathrm{hr}$, reaching about $10 \%$ of the control area at $72 \mathrm{hr}$ (Fig. 3B).

To examine whether these effects were due to active MMP-2, we blocked MMP-2 by adding a specific inhibitor (0 and $2.5 \mu \mathrm{M}$ ) to the ConA-containing medium. As expected, the appearance of fibrillin- 1 and fibrillin-2 in the MMP-2 inhibitor I-treated culture was almost the same as that in the control culture (Fig. 4). Therefore, we confirmed that the degradation of fibrillin-1 and fibrillin-2 had been due to MMP-2 activated by ConA. The appearance of the control culture (containing less than $0.2 \%$ DMSO) was similar to that of the non-treated culture we had described previously [29, 31] (not shown).

\section{Discussion}

In the present study, we have demonstrated for the first time that active MMP-2 degrades the network of oxytalan fibers produced by HNPCEC and PDL fibroblasts, and that the patterns of fibrillin- 1 and fibrillin-2 degradation in the two cell types are completely opposite. That is, fibrillin-2 is preferentially degraded in oxytalan fibers of cultured HNPCEC, whereas fibrillin-1 is preferentially degraded in oxytalan fibers of cultured PDL fibroblasts. The appearance of fibrillin-1- and fibrillin-2-positive fibers did not change under control cultures in DMEM without ConA within 72 $\mathrm{hr}$ (not shown). Therefore, the change of appearance of fibrillin-1 and fibrillin-2 is thought to reflect the degradation of each molecule. The two molecules interact to form homotypic and heterotypic dimers in microfibrils $[5,16]$. Fibrillin-2 is found preferentially in elastic tissues, such as elastic cartilage, the aorta and the lung [33], whereas fibrillin-1 is expressed in load-bearing tissues such as the aortic adventitia and skin [34]. Although fibrillin-1 and fibrillin-2 share some overlapping functions [4], our results may reflect the specific role of each fibrillin molecule in 
(A)

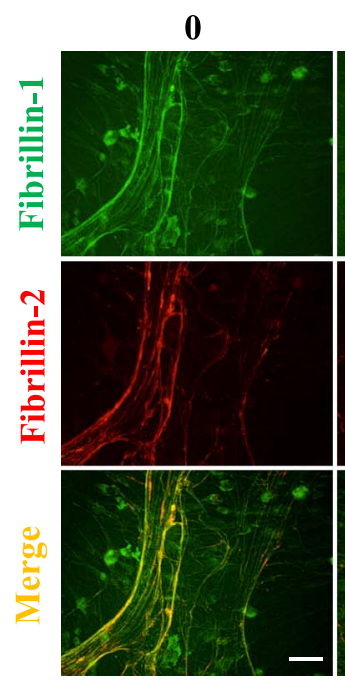

24

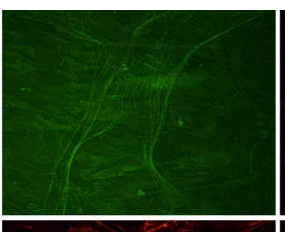

48

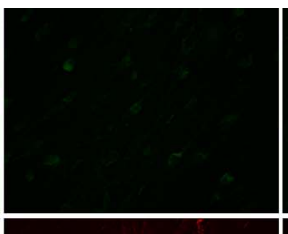

72 hr

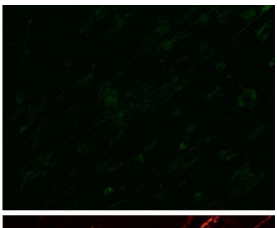

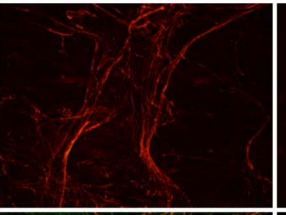
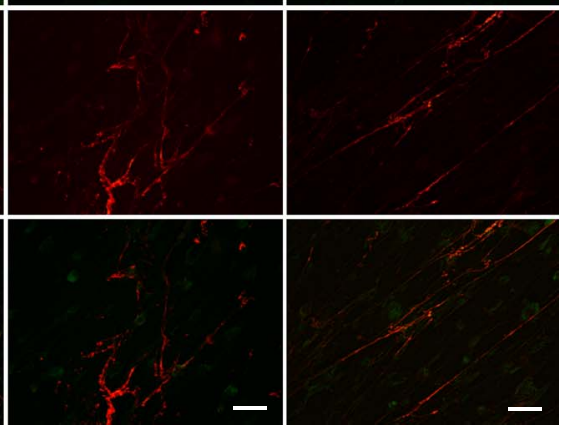

(B)

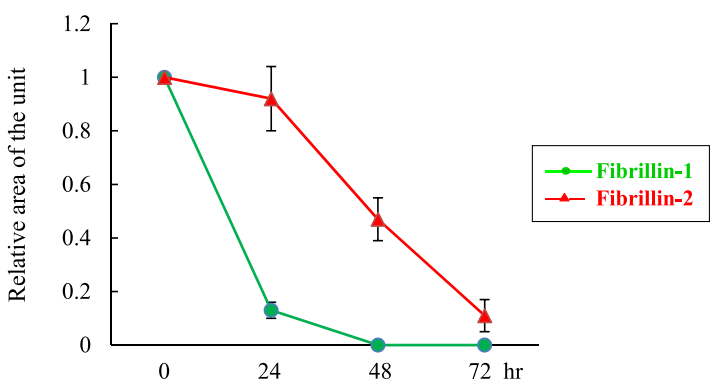

Fig. 3. Degradation of fibrillin-1 and fibrillin-2 by active MMP-2. (A) Double immunofluorescence for fibrillin-1 (upper panels) and fibrillin-2 (middle panels) in cultures of human periodontal ligament fibroblasts. Human periodontal ligament fibroblasts were cultured for 7 days, and then $50 \mathrm{ng} / \mathrm{ml} \mathrm{ConA} \mathrm{was} \mathrm{added} \mathrm{to} \mathrm{the} \mathrm{medium} \mathrm{for} \mathrm{an} \mathrm{additional} 72 \mathrm{hr}$. Cells were then simultaneously labeled for fibrillin-1 (green) (upper panels), fibrillin-2 (red) (middle panels), and superimposition of both labels (lower panels) at 0, 24, 48 and $72 \mathrm{hr}$. Bar=20 $\mu \mathrm{m}$. (B) Planimetry analysis of the positive signals was performed using the Image J program. The relative area of positive signals at $0 \mathrm{hr}$ was obtained using Image $\mathrm{J}$ with the control set as 1 . Data represent the mean \pm S.D. (standard deviation) of four independent experimental determinations.

the eye and PDL.

The human ciliary zonule, with a diameter of 20-30 $\mu \mathrm{m}$, suspends the lens from the ciliary body, thereby effectively transmitting contraction of the ciliary body muscle to the lens for focusing. We recently demonstrated in vitro that fibrillin- 2 contributes to the formation of thick oxytalan fibers during development of the ciliary zonule [31]. The ciliary zonule is thought to undergo continuous remodeling because its location of attachment to the lens changes with age [21]. The mechanism of degradation of the ciliary zonule under physiological conditions has been unclear. HNPCEC are known to express MMP-1, -2, -3 and $-9[11,20]$. On the other hand, lens epithelial cells are reported to express various MMPs [13]. Lens epithelial cells do not contribute to the production of the ciliary zonule because they lack expression of fibrillin-1 and fibrillin-2 $[12,24]$. However, they may control the remodeling of the lens and contribute to zonule degradation. Further investigations are needed to elucidate the role of lens epithelial cells in relation to the remodeling of the ciliary zonule.

MMP-2 has been considered a major enzyme involved in the turnover of the extracellular matrix [6]. This study represents the first in vitro demonstration that ConA-activated MMP-2 degrades oxytalan fibers. ConA is known to activate proMMP-2 through membrane type 1 MMP [22]. In our culture system, HNPCEC and PDL fibroblasts form an oxytalan fiber network within 7 days, when secreted MMP-2 is still in the inactive pro-MMP-2 form. Therefore, we added ConA to induce the activation of MMP-2 in order to examine the sensitivity of fibrillin-1 and fibrillin-2 to MMP-2. Fibrillin-1 and fibrillin-2, the main structural components of oxytalan fibers, are both substrates for MMP-2 [1, 10]. The turnover of elastic system fibers is thought to be slow [17], and the details of the physiological degradation of oxytalan fibers have never been reported previously. We added a MMP-2-specific inhibitor to confirm whether degradation of fibrillin-1 and fibrillin-2 was due to MMP-2 itself. The contrasting patterns of degradation of fibrillin-1 and fibrillin-2 in the 


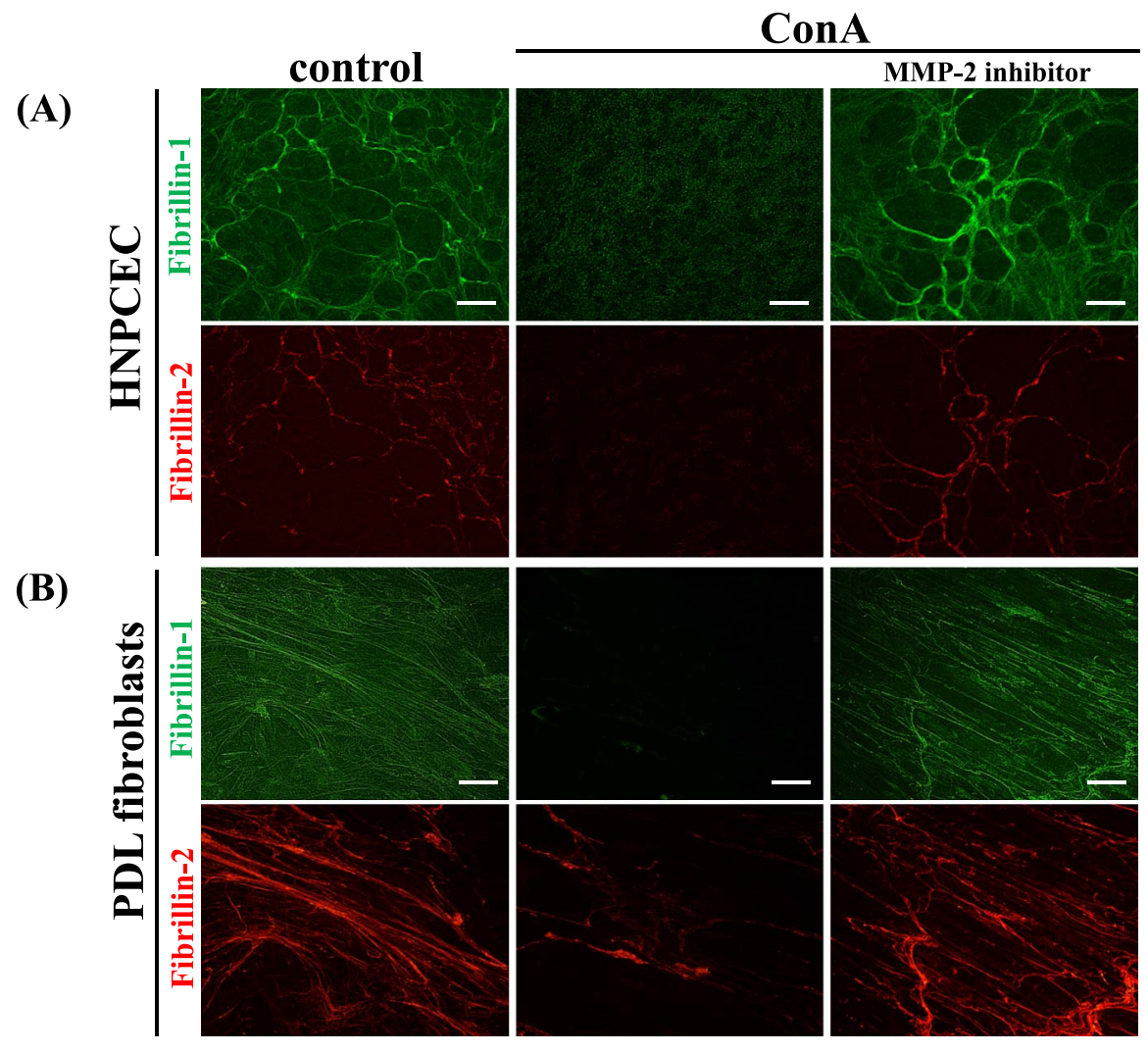

Fig. 4. Evidence that MMP-2 degrades fibrillin-1 and fibrillin-2. Human non-pigmented ciliary epithelial cells (A) and periodontal ligament fibroblasts (B) were cultured for 7 days, and then $50 \mathrm{ng} / \mathrm{ml} \mathrm{ConA} \mathrm{was} \mathrm{added} \mathrm{to} \mathrm{the} \mathrm{medium} \mathrm{for} \mathrm{an} \mathrm{additional} 72 \mathrm{hr}$. In some cultures, $2.5 \mu \mathrm{M}$ MMP-2 inhibitor I as well as $50 \mathrm{ng} / \mathrm{ml}$ ConA was added to the medium. After $72 \mathrm{hr}$, the cells were simultaneously labeled for fibrillin-1 (green) (upper panels) and fibrillin-2 (red). The left panel is the control (non-treated) culture, the middle panel is the ConA-treated culture, and the right panel is the culture treated with both ConA and MMP-2 inhibitor I. Bar $=20 \mu \mathrm{m}$.

zonule and PDL under experimentally MMP-2-activated conditions are of considerable interest since they may reflect the characteristics of each oxytalan fiber in the two tissues.

In summary, by means of immunofluorescence analysis, we have demonstrated that HNPCEC and PDL fibroblasts both form fibrillin-1 and fibrillin-2 networks, but that these show contrasting patterns of degradation by activated MMP-2. This may reflect the sensitivity of fibrillin-1 and fibrillin-2 of the two different types of oxytalan fibers in the eye and PDL against MMP-2. Further quantitative analysis will be needed for comparison of the two tissues.

\section{References}

1. Ashworth, J. L., Murphy, G., Rock, M. J., Sherratt, M. J., Shapiro, S. D., Shuttleworth, C. A. and Kielty, C. M. (1999) Fibrillin degradation by matrix metalloproteinases: implications for connective tissue remodelling. Biochem. J. 340; 171-181.

2. Böck, P. and Stockinger, L. (1984) Light and electron microscopic identification of elastic, elaunin and oxytalan fibers in human tracheal and bronchial mucosa. Anat. Embryol. (Berl) 170; $145-153$.

3. Cain, S. A., Morgan, A., Sherratt, M. J., Ball, S. G., Shuttleworth,
C. A. and Kielty, C. M. (2006) Proteomic analysis of fibrillin-rich microfibrils. Proteomics 6; 111-122.

4. Carta, L., Pereira, L., Arteaga-Solis, E., Lee-Arteaga, S. Y., Lenart, B., Starcher, B., Merkel, C. A., Sukoyan, M., Kerkis, A., Hazeki, N., Keene, D. R., Sakai, L. Y. and Ramirez, F. (2006) Fibrillins 1 and 2 perform partially overlapping functions during aortic development. J. Biol. Chem. 281; 8016-8023.

5. Charbonneau, N. L., Dzamba, B. J., Ono, R. N., Keene, D. R., Corson, G. M., Reinhardt, D. P. and Sakai, L. Y. (2003) Fibrillins can co-assemble in fibrils, but fibrillin fibril composition displays cell-specific differences. J. Biol. Chem. 278; 2740-2749.

6. Creemers, L. B., Jansen, I. D., Docherty, A. J., Reynolds, J. J., Beertsen, W. and Everts, V. (1998) Gelatinase A (MMP-2) and cysteine proteinases are essential for the degradation of collagen in soft connective tissue. Matrix Biol. 17; 35-46.

7. Fullmer, H. M. and Lillie, R. D. (1958) The oxytalan fiber: a previously undescribed connective tissue fiber. J. Histochem. Cytochem. 6; 425-430.

8. Hanssen, E., Franc, S. and Garrone, R. (2001) Synthesis and structural organization of zonular fibers during development and aging. Matrix Biol. 20; 77-85.

9. Hens, J., Dann, P., Hiremath, M., Pan, T. C., Chodosh, L. and Wysolmerski, J. (2009) Analysis of gene expression in PTHrP-/mammary buds supports a role for BMP signaling and MMP2 in the initiation of ductal morphogenesis. Dev. Dyn. 238; 27132724.

10. Hindson, V. J., Ashworth, J. L., Rock, M. J., Cunliffe, S., 
Shuttleworth, C. A. and Kielty, C. M. (1999) Fibrillin degradation by matrix metalloproteinases: identification of amino- and carboxy-terminal cleavage sites. FEBS Lett. 452; 195-198.

11. Hinz, B., Rosch, S., Ramer, R., Tamm, E. R. and Brune, K. (2005) Latanoprost induces matrix metalloproteinase-1 expression in human nonpigmented ciliary epithelial cells through a cyclooxygenase-2-dependent mechanism. FASEB J. 19; 19291931.

12. Hiraoka, M., Inoue, K., Ohtaka-Maruyama, C., Ohsako, S., Kojima, N., Senoo, H. and Takada, M. (2010) Intracapsular organization of ciliary zonules in monkey eyes. Anat. Rec. (Hoboken) 293; 1797-1804

13. Hodgkinson, L. M., Duncan, G., Wang, L., Pennington, C. J., Edwards, D. R. and Wormstone, I. M. (2007) MMP and TIMP expression in quiescent, dividing, and differentiating human lens cells. Invest. Ophthalmol. Vis. Sci. 48; 4192-4199.

14. Kielty, C. M., Baldock, C., Lee, D., Rock, M. J., Ashworth, J. L. and Shuttleworth, C. A. (2002) Fibrillin: from microfibril assembly to biomechanical function. Philos. Trans. R. Soc. Lond. B Biol. Sci. 357; 207-217.

15. Lemaitre, V. and D'Armiento, J. (2006) Matrix metalloproteinases in development and disease. Birth Defects. Res. C. Embryo. Today 78; 1-10.

16. Lin, G., Tiedemann, K., Vollbrandt, T., Peters, H., Batge, B., Brinckmann, J. and Reinhardt, D. P. (2002) Homo- and heterotypic fibrillin-1 and -2 interactions constitute the basis for the assembly of microfibrils. J. Biol. Chem. 277; 50795-50804.

17. Mecham, R. P. and Davis, E. C. (1994) Elastic fiber structure and assembly. In "Extracellular matrix assembly and structure" ed. by P. D. Yurchenco, D. E. Birk and R. P. Mecham, Academic Press, New York, pp. 281-314.

18. Nakashima, K., Tsuruga, E., Hisanaga, Y., Ishikawa, H. and Sawa, Y. (2009) Stretching stimulates fibulin-5 expression and controls microfibril bundles in human periodontal ligament cells. J. Periodontal Res. 44; 622-627.

19. Raviola, G. (1971) The fine structure of the ciliary zonule and ciliary epithelium. With special regard to the organization and insertion of the zonular fibrils. Invest. Ophthalmol. 10; 851-869.

20. Rosch, S., Ramer, R., Brune, K. and Hinz, B. (2006) R(+)methanandamide and other cannabinoids induce the expression of cyclooxygenase-2 and matrix metalloproteinases in human nonpigmented ciliary epithelial cells. J. Pharmacol. Exp. Ther. $316 ; 1219-1228$

21. Sakabe, I., Oshika, T., Lim, S. J. and Apple, D. J. (1998) Anterior shift of zonular insertion onto the anterior surface of human crystalline lens with age. Ophthalmology 105; 295-299.

22. Sato, H., Takino, T., Okada, Y., Cao, J., Shinagawa, A., Yamamoto, E. and Seiki, M. (1994) A matrix metalloproteinase expressed on the surface of invasive tumour cells. Nature 370 ; $61-65$.
23. Sawada, T. (2010) Ultrastructural localization of fibrillin-1 and fibrillin-2 in oxytalan fibers in periodontal ligament of Japanese Macaca fuscata monkey. J. Mol. Histol. 41; 225-231.

24. Shi, Y., Tu, Y., De Maria, A., Mecham, R. P. and Bassnett, S. (2013) Development, composition, and structural arrangements of the ciliary zonule of the mouse. Invest. Ophthalmol. Vis. Sci. 54; 2504-2515.

25. Sims, M. R. (1973) Oxytalan fiber system of molars in the mouse mandible. J. Dent. Res. 52; 797-802.

26. Sugawara, Y., Sawada, T., Inoue, S., Shibayama, K. and Yanagisawa, T. (2010) Immunohistochemical localization of elastin, fibrillins and microfibril-associated glycoprotein-1 in the developing periodontal ligament of the rat molar. J. Periodontal Res. $45 ; 52-59$.

27. Tsuruga, E., Irie, K., Sakakura, Y. and Yajima, T. (2002) Expression of fibrillins and tropoelastin by human gingival and periodontal ligament fibroblasts in vitro. J. Periodontal Res. 37; 23-28.

28. Tsuruga, E., Irie, K. and Yajima, T. (2002) Gene expression and accumulation of fibrillin-1, fibrillin-2, and tropoelastin in cultured periodontal fibroblasts. J. Dent. Res. 81;771-775.

29. Tsuruga, E., Nakashima, K., Ishikawa, H., Yajima, T. and Sawa, Y. (2009) Stretching modulates oxytalan fibers in human periodontal ligament cells. J. Periodont. Res. 44; 170-174.

30. Vu, T. H. and Werb, Z. (2000) Matrix metalloproteinases: effectors of development and normal physiology. Genes. Dev. 14; 2123-2133.

31. Yamanouchi, K., Tsuruga, E., Oka, K., Sawa, Y. and Ishikawa, H. (2012) Fibrillin-1 and fibrillin-2 are essential for formation of thick oxytalan fibers in human nonpigmented ciliary epithelial cells in vitro. Connect. Tissue Res. 53; 14-20.

32. Yamauchi, Y., Tsuruga, E., Nakashima, K., Sawa, Y. and Ishikawa, H. (2010) Fibulin-4 and -5, but not fibulin-2, are associated with tropoelastin deposition in elastin-producing cell culture. Acta Histochem. Cytochem. 43; 131-138.

33. Zhang, H., Apfelroth, S. D., Hu, W., Davis, E. C., Sanguineti, C., Bonadio, J., Mecham, R. P. and Ramirez, F. (1994) Structure and expression of fibrillin-2, a novel microfibrillar component preferentially located in elastic matrices. J. Cell Biol. 124; 855863.

34. Zhang, H., Hu, W. and Ramirez, F. (1995) Developmental expression of fibrillin genes suggests heterogeneity of extracellular microfibrils. J. Cell Biol. 129; 1165-1176.

This is an open access article distributed under the Creative Commons Attribution License, which permits unrestricted use, distribution, and reproduction in any medium, provided the original work is properly cited. 